\title{
Recombinant luciferase-expressing human cytomegalovirus (CMV) for evaluation of CMV inhibitors
}

\author{
Ran He ${ }^{1}$, Gordon Sandford², Gary S Hayward², William H Burns², Gary H Posner ${ }^{3}$, Michael Forman ${ }^{4}$, Ravit Arav-Boger ${ }^{1 *}$
}

\begin{abstract}
Recombinant Towne CMV expressing luciferase under the control of CMV-DNA polymerase (POL) or the late pp28 (UL99) promoters were evaluated for potential application in high-throughput screening of anti-viral compounds. POL-and pp28-luciferase displayed maximal expression 48 and 72 hours post infection, respectively. The pp28luciferase virus achieved a wider dynamic range of luciferase expression (6-7 logs) and was selected for testing of inhibition by five anti-viral compounds. Luciferase expression highly correlated with plaque reduction and real-time PCR. The pp28-luciferase reporter system is rapid, reproducible, and highly sensitive. It may be applied to screening of novel anti-CMV compounds.
\end{abstract}

\section{Background}

Infection with Cytomegalovirus (CMV) continues to be a major threat in organ transplant recipients and congenitally-infected children $[1,2]$. Although existing systemic therapies are effective in suppressing virus replication, serious side effects and the emergence of resistant viral strains pose significant treatment dilemmas [3]. The need to identify and develop new antiCMV compounds coincides with the advancement of rapid, sensitive, and high-throughput methods for screening of lead compounds. While the plaque reduction assay remains the gold-standard for screening of anti-viral compounds, new techniques based on recombinant DNA technology and highly sensitive molecular assays have recently been suggested as alternatives [4-6]. Real-time PCR, the standard of care in the management of CMV disease in high- risk patient populations, may also provide a sensitive tool for drug screening [7-12]

In earlier studies, a series of chloramphenicol acetyl transferase (CAT) recombinants expressing CAT under the control of UL54 (DNA polymerase, POL) or UL99 (pp28) promoters were constructed. The expression of $\mathrm{CAT}$ in infected cells highly mimicked the expression pattern of the endogenous UL54 and UL99 $[4,13]$. Thus,

\footnotetext{
* Correspondence: boger@jhmi.edu

'Department of Pediatrics, Johns Hopkins University School of Medicine, Baltimore, MD, USA

Full list of author information is available at the end of the article
}

these two gene promoters were selected to construct luciferase-recombinant CMV for quatification of CMV replication in a rapid and reproducuble way. We report on the evaluation of two luciferase recombinant viruses (pp28 and POL) and the correlation of the pp28-luciferase system with plaque reduction and real-time PCR in evaluation of CMV inhibition by anti-CMV compounds.

\section{Methods}

\section{Construction of luciferase viruses}

Recombinant CMV based on the laboratory-adapted strain, Towne, was constructed by homologous recombination in transfected-infected cells. A $\beta$-galactosidase $(\beta$ -gal)-expressing Towne virus was first constructed using an intergenic insertion site between US9 and US10. Prior studies in which a $\beta$-glucuronidase expression cassette was inserted in this intergenic region of the laboratory-adapted AD169 virus revealed no alteration in expected transcription from this region $[4,14,15]$. The recombinant was genetically stable and exhibited normal in-vitro growth characteristics. The transfer vector, $\mathrm{pT}$, was constructed from pRL120 which contains the Towne virus HindIII T fragment [16]. A $2.0 \mathrm{~kb}$ BamHIApaI subfragment containing US9 was ligated into pGEM11z (Promega, Madison, WI) and the adjacent 1.3 kb ApaI-ApaI fragment containing US10 was isolated from agarose gels and ligated into the ApaI site. DNA sequencing confirmed the correct orientation of this 
fragment. The BstEII site, which lies midway between the US9 and US10 genes, was used as the insertion site for the $\beta$-gal expression cassette containing an SV40 promoter and polyA signal (pSV $\beta$ from Clontech, Mountain View, CA). DNA extracted from human foreskin fibroblasts (HFF) infected with Towne virus and linearized transfer vector containing the expression cassette were coprecipitated onto subconfluent HFF cultures by the calcium phosphate method [17], followed by a 2 min shock with $20 \%$ Dimethyl sulfoxide (DMSO) in Minimum Essential Medium (MEM) 4 to 6 hrs later. Virus from cultures developing cytopathic effects was passed onto fresh HFF cultures, and examined for $\beta$-galactosidase activity. Recombinant virus, designated T242, was isolated from positive cultures by limiting dilution in 96 well plates of HFF and selection of $\beta$-gal positive wells at the highest dilutions.

To produce a recombinant virus expressing the luciferase reporter gene under the control of either the promoter of an early gene (POL, UL54) or a late gene (pp28, UL99), the expression cassette of luciferase was substituted for the $\beta$-gal cassette using the same transfer vector (pT). Expression cassettes of luciferase under the control of $P O L$ - or pp28-promoter were constructed by cloning the PCR products of the upstream $500 \mathrm{bp}$ of DNA polymerase or 350 bp of pp 28 genes and ligating them into the 5' position of the luciferase coding region. These expression cassettes were then ligated into the blunted BstEII site of the pT transfer vector, linearized and used in coprecipitation experiments with the DNA of HFF cells infected with T242. Successful replacement of the $\beta$-gal expression cassette by the luciferase expression cassettes with loss of $\beta$-gal expression and acquisition of luciferase expression as phenotypic markers facilitated isolation of the desired recombinants. Several PCR sequencing reactions confirmed the correct position and orientation of the luciferase reporter gene. The following primers were used: primer 1- US09 forward 5'-ACCTTGAAATGGGTCGCGCTCCGCT-3', primer 2- luciferase forward-5'-ACAAGGATATGGGCTCACTGAGACT-3', primer 3: luciferase reverse 5'-AGTCTCAGTGAGCCCATATCCTTGT-3', and primer 4- US10 reverse- 5'-GCTATCGTCGCCGGAAGGAAACCGA -3'.

\section{Cell Culture and virus infection}

HFF and human lung fibroblasts (HEL) (ATCC, CRL2088 and CCL-137, respectively) were propagated in Dulbecco's Modified Eagle Medium (DMEM) containing $10 \%$ fetal bovine serum (FBS) and used for infections with the luciferase viruses. For assays other than plaque reduction, $4 \times 10^{4} \mathrm{HFF}$ cells were seeded in each well of 24-well plate one day prior to infection. Luciferase viruses were used for infections with multiplicity of infection (MOI) of 1.0 as previously described [18].
After 90 minutes adsorption, virus was removed, and $0.5 \mathrm{ml}$ of media containing specified concentrations of antiviral compounds was added. Infected non-treated cells were used as positive controls; non-infected cell lysates were used as negative controls.

\section{Luciferase Assay}

HFF cells were collected and lysed with Wizard ${ }^{\circledR}$ SV Lysis Buffer (Promega, Madison, WI). The lysates were assayed for luciferase and cell viability using an automated luminescent assay (Promega, Madison, WI), and CellTiter-Glo luminescent cell viability assay kit, respectively, on GloMax ${ }^{\circledR}$-Multi+ Detection System (Promega, Madison, WI) according to manufacturer's instructions.

\section{Plaque reduction assay}

HEL cells were seeded at $3 \times 10^{5}$ cells per well in twelve-well plates and were infected 24 hours later with the pp28-luciferase CMV at $60 \mathrm{PFU} /$ well. Following 90 minutes adsorption, the medium was aspirated from the wells, and fresh medium containing selected drug dilutions of ganciclovir (GCV), Foscarnet (FOS), Cycloheximide (CHX), artesunate (ART), dimer sulfone carbamate [19] and $0.5 \%$ of carboxymethyl-cellulose were added into triplicate wells. After incubation at $37^{\circ} \mathrm{C}$ for 8 days, the overlay was removed, and the monolayer was stained with crystal violet. Plaques were counted microscopically under low power $(40 \times)$. Drug effects were calculated as the percentage of reduction in number of plaques in the presence of each drug concentration to the number observed in the absence of drug.

\section{Virus yield reduction assay}

HFF were infected with the original Towne virus or pp28/POL- luciferase virus at an MOI of 0.1. Culture supernatants were collected every two days until day 10 post infection and frozen at $-80^{\circ} \mathrm{C}$. Collected samples were thawed and used for titration of infectious virus by the plaque assay.

\section{Real-time PCR}

The quantitative CMV real-time PCR assay is based on detection of a 151bp region from the highly conserved US17 gene [20]. The limit of detection of the assay is 100 copies $/ \mathrm{mL}$ (3.0 copies/reaction), and the measureable range is $2.4-8.0 \log _{10}$ copies $/ \mathrm{mL}$. The PCR was performed using a total reaction volume $50 \mu \mathrm{L}$. This included $25 \mu \mathrm{L}$ of TaqMan 2X Universal PCR Master Mix (Applied Biosystems, Foster City, CA), $1.5 \mu \mathrm{L}$ each of $10 \mu \mathrm{M}$ primers, $1 \mu \mathrm{L}$ of $10 \mu \mathrm{M}$ FAM-labeled probe, $11 \mu \mathrm{L}$ of $\mathrm{dH}_{2} \mathrm{O}$, and $10 \mu \mathrm{l}$ of template. Amplification was performed on a 7500 Real-Time PCR System (Applied Biosystems, Foster City, CA). PCR conditions were: $50^{\circ} \mathrm{C}$ for $2 \mathrm{~min}, 95^{\circ} \mathrm{C}$ for $10 \mathrm{~min}, 40$ cycles of $95^{\circ} \mathrm{C}$ for $15 \mathrm{~s}$ 
and $60^{\circ} \mathrm{C}$ for $60 \mathrm{~s}$. Quantification standards were prepared by cloning the US17 amplicon in the $\mathrm{pCR}^{\circledR} 2.1$ TOPO $^{\circledR}$ plasmid vector (Invitrogen, Carlsbad, CA). Serial 10 -fold dilutions of plasmid from 7.0 to $1.0 \log _{10}$ copies/reaction were included with each assay and used to establish a standard curve. Assay controls included quantified CMV AD169 DNA (Advanced Biotechnologies Inc.) and quantified Towne CMV at 3.0 and 5.0 $\log _{10}$ copies/mL. Quantitative CMV data were expressed as viral DNA copies per milliliter.

\section{Antiviral compounds}

GCV, sodium phosphonoformate (FOS) and cycloheximide (CHX) were obtained from Sigma-Aldrich (St. Louis, MO). Artemisinin derivatives, monomeric trioxane artesunate (ART) and trioxane dimer sulfone carbamate were synthesized at Johns Hopkins University (GHP), and their structural details have been provided elsewhere [18].

\section{Results}

\section{Luciferase constructs}

Two luciferase expressing viruses were constructed with the Towne CMV strain (Figure 1A). A recombinant $\beta$ galactosidase ( $\beta$-gal) CMV strain was first prepared as a backbone for luciferase CMV. Recombinant $\beta$-gal virus was isolated from positive cultures. This virus was used in a second-round DNA recombination to generate two luciferase-reporter CMV viruses: the luciferase gene being under the control of either UL54 (POL) or UL99 (pp28) promoters. Successful recombinants were isolated by loss of $\beta$-gal activity and the expression of luciferase protein. The loss of the $\beta$-gal gene and acquisition of the luciferase gene in the expected location was confirmed by DNA sequencing (Genebank submission ID: 1420040, sequences are also available in Additional file 1). Insertion at the specific sites was verified by PCR sequencing (Figure 1B).

\section{Comparison of luciferase expression by the two viral constructs}

The recombinant viruses were expected to express luciferase at different stages of virus replication. The early gene UL54 $(P O L)$ is expressed within the first 24 hours post infection (hpi), usually later than 12 hpi [21]; whereas the true late UL99 (pp28) gene is expressed only at or after $48 \mathrm{hpi}$. Luciferase expression by $P O L-$ and pp28-luciferase was quantified in cell lysates at 12, $24,36,48,72 \mathrm{hpi}$, and at 36, 48, 72 and $96 \mathrm{hpi}$, respectively (Figure 2). Using the same cell conditions, infectivity, and luciferase assay system, peak luciferase activities measured with pp28-luciferase were 20 fold higher than those measured with $P O L$-luciferase. The peak activity of pp28-luciferase was reached at $72 \mathrm{hpi}$,

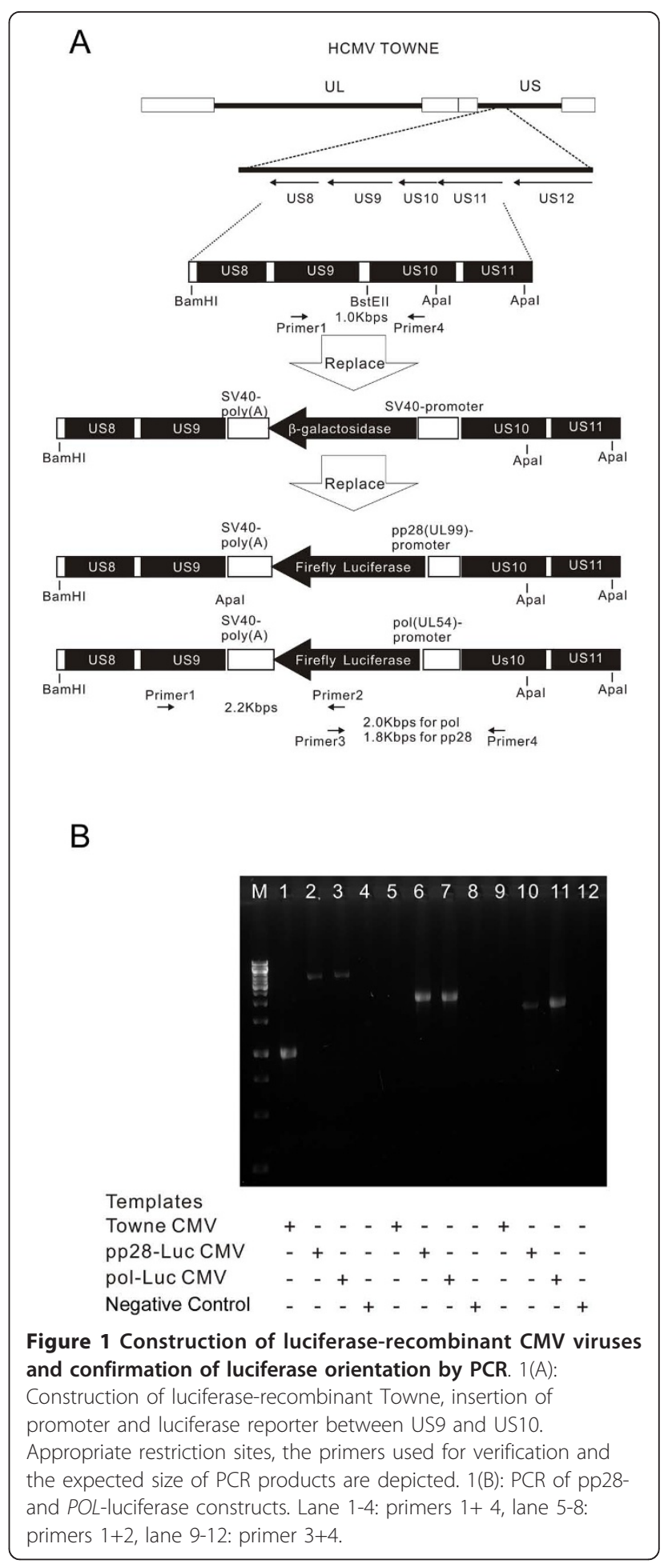

followed by a plateau towards 96 hpi. POL-luciferase reached its maximum expression at 48 hours post infection. The dynamic range of the luciferase assay using pp28-luciferase and POL-luciferase was $50-5 \times 10^{6}$, and $50-6 \times 10^{4}$ respectively; therefore the pp28-luciferase virus was used in subsequent experiments. 


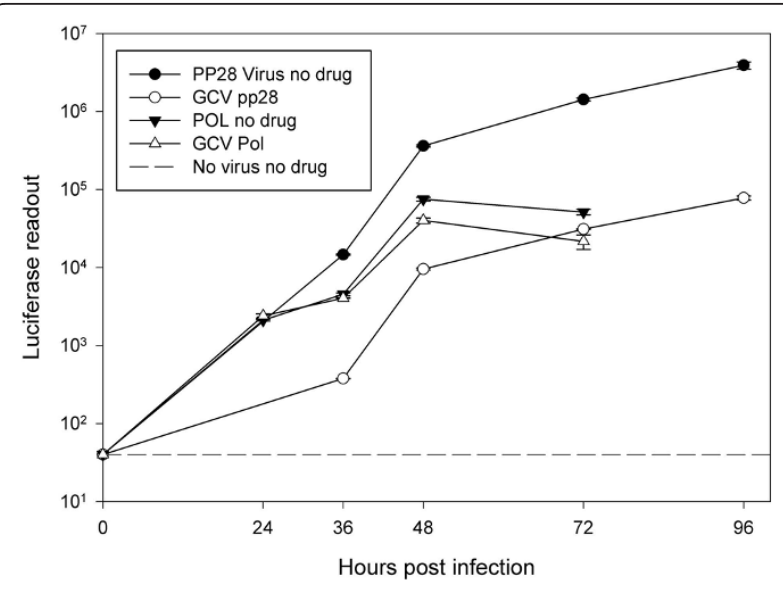

Figure 2 Timing and expression pattern of pp28-and POLluciferase CMV. Luciferase expression was determined in celllysates at indicated time points following infection with pp28- or POL-luciferase with and without treatment with GCV $(30 \mu \mathrm{M})$. Y axislog scale of luciferase read out; $X$ axis- time points in hours.

\section{Growth Characteristics of pp28-luciferase and the parent Towne virus}

We evaluated whether insertion of the recombination cassette affected the growth kinetics and production of infectious progeny. The parent Towne virus, pp28- and pol-luciferase Towne viruses were grown in HFF and the production of infectious progeny was determined every two days during 10 day course post infection. The

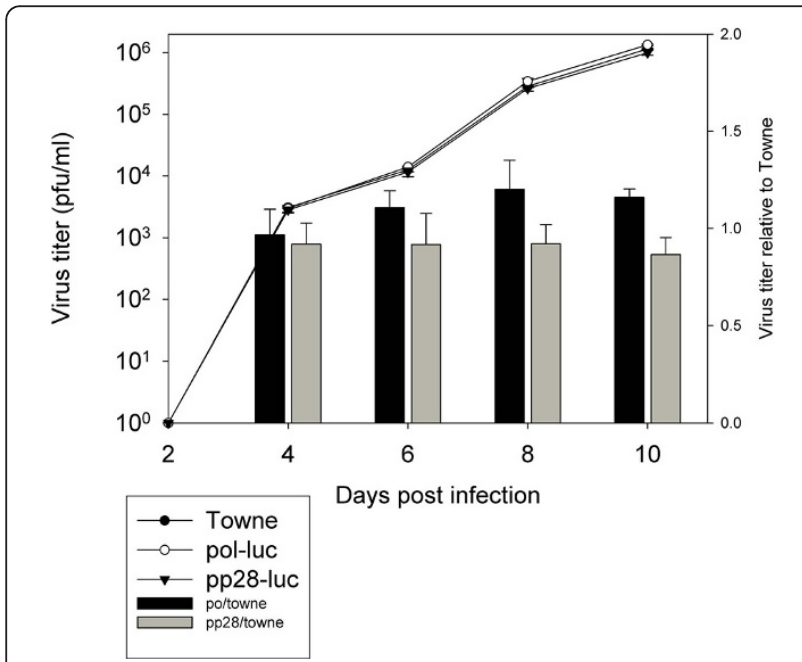

Figure 3 Growth characteristics of Towne, pp28-and POLluciferase Towne viruses. The production of virus progeny was determined in HFF infected with the original Towne virus, and recombinant $\mathrm{pp} 28$ - or $\mathrm{POL}-\mathrm{luciferase}$ virus at an $\mathrm{MOI}$ of 0.1 . Culture supernatants were collected at the indicated days and used for titration of infectious virus by the plaque assay. Y-axis on the left indicates growth of progeny viruses in log scale, $Y$-axis on the right indicated relative virus kinetics of the recombinant viruses as compared to the parent Towne strain. growth characteristics of the viruses were similar (Figure 3). A marked increase in virus production was observed starting 2 days post infection, and growth kinetics was similar to previous reports [22]

\section{Correlation of plaque reduction and luciferase expression} Parallel experiments were conducted using the same MOI of pp28-luciferase CMV with and without antiCMV compounds (GCV, FOS, ART, dimer sulfone carbamate, $\mathrm{CHX}$ ). The relative number of plaques counted 10 days post infection was compared to relative luciferase activities assayed $72 \mathrm{hpi}$ (Figure 4 Table 1). The drug concentration inhibiting 50\% virus replication $\left(E_{50}\right)$ by plaque reduction and luciferase expression was determined for each compound. For all five compounds a high correlation was observed between plaque reduction and luciferase expression (Figure 4). Data obtained with the plaque reduction assay were similar to previous reports (Table 1).

\section{Inhibition of luciferase expression and DNA replication by} dimer sulfone carbamate and GCV

The supernatants from infected-treated and infectednon treated cells were used for real-time PCR at day 3 . However, the test was not sensitive enough to detect differences between the treatment conditions (data not shown). Therefore, luciferase activity was compared with real-time PCR from supernatants of infected cells 6 days post infection. A high correlation was found between luciferase expression, and DNA copy number (Figure 5).

\section{Discussion}

We report on a highly sensitive and objective luciferase reporter assay for determination of CMV inhibition by anti-viral agents. The assay, based on pp28-luciferase recombinant CMV, can be performed 72 hpi and drug treatment, has a large dynamic range of 6-7 logs, and is highly reproducible. Our work also reveals a high degree of correlation between late gene (luciferase) expression and plaque enumeration further confirming the potential use of this assay in screening of anti-viral activities.

The susceptibility of CMV strains, laboratory-adapted and clinical isolates, to anti-CMV compounds has traditionally been evaluated by the classic plaque assay [23]. Although this assay best reflects viral infectivity, or the biological behavior of CMV, it suffers from several drawbacks. The assay is time consuming; results are usually available 8-21 days after infection depending on the virus strain used, and counting of plaques is labor intensive. Another disadvantage of the plaque assay is that the amount of viral replication within a single cell cannot always be determined. Not infrequently, the endpoint of the test shows enlarged cells (CPE) without spread of the virus to adjacent cells (plaque). 


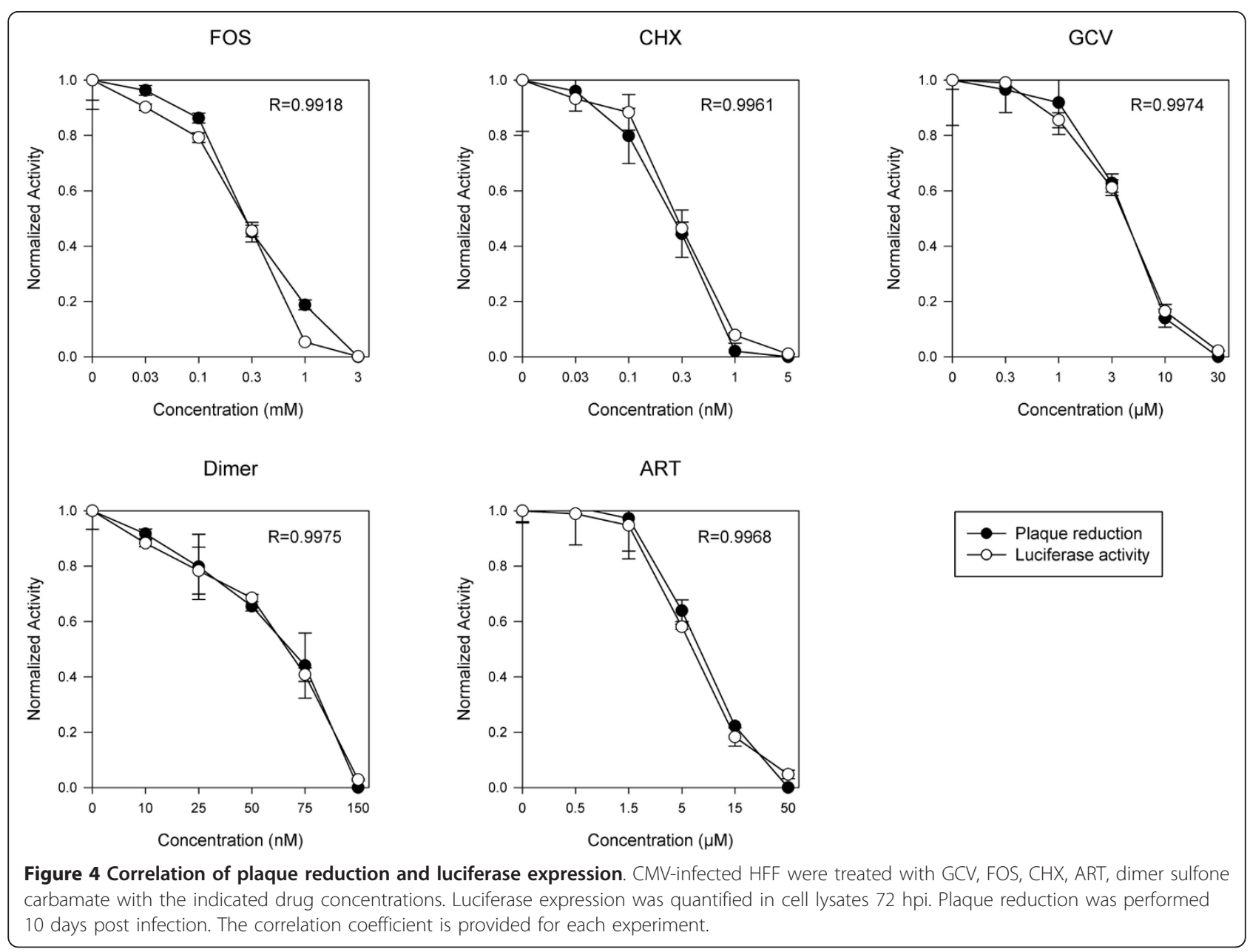

Recombinant viruses carrying different reporter genes have been developed as alternative methods to overcome some of the limitations of the plaque assay. A recombinant CMV expressing $\beta$-galactosidase under the control of the major immediate early promoter was used in a 96-well assay [24]. Although the assay was sensitive and rapid, background $\beta$-galactosidase activity was observed secondary to its expression under the control of an immediate early gene during the initial infection. A secreted alkaline phosphatase (SEAP) reporter gene driven by the CMV major immediate early promoter was inserted at the US6 gene [25]. Reduction in SEAP activity under drug treatment was used to determine drug sensitivity. Results of transferring specific mutations in UL97 or POL were compared with results obtained using traditional phenotyping assays. The assay was validated for approved CMV drugs (GCV, FOS, and CDV) that target the CMV DNA polymerase. The open reading frame between US9 and US10 has been used to construct several recombinant CMV strains $[4,5,26]$. For example, a GFP- reporter system

Table 1 Inhibition of pp28-luciferase by anti-CMV compounds using plaque reduction or luciferase assay

\begin{tabular}{lccc}
\hline Compound & Plaque Reduction $\mathrm{EC}_{\mathbf{5 0}}(\boldsymbol{\mu} \mathrm{M})$ & Luciferase & Reference \\
\hline FOS & $328+/-28$ & $268+/-20$ & {$[28]$} \\
Dimer Sulfone Carbamate & $0.067+/-0.011$ & $0.066+/-0.004$ & {$[18]$} \\
ART & $8.03+/-0.55$ & $6.74+/-0.38$ & {$[29]$} \\
GCV & $4.39+/-0.39$ & $4.23+/-0.27$ & {$[30]$} \\
CHX & $0.262+/-0.067$ & $0.299+/-0.036$ & $\mathrm{NA}$ \\
\hline
\end{tabular}

$\mathrm{EC}_{50}$ was determined by plaque reduction assay or luciferase expression in pp28-luciferase CMV infected HFF cells. Reported values represent the means \pm standard deviations (SD) of data derived from at least three independent experiments performed in duplicate. Historical controls are provided for $\mathrm{EC}_{50}$ values (reference column). 


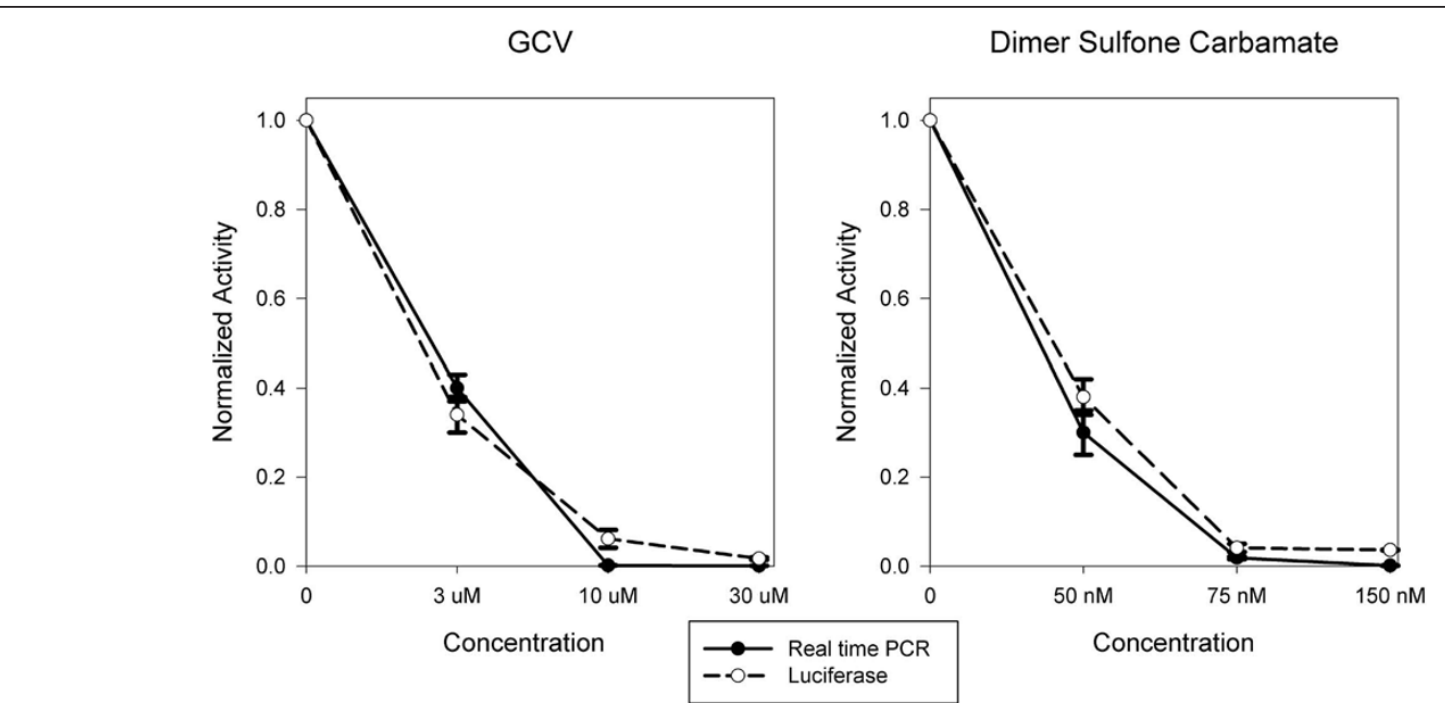

Figure 5 Luciferase expression and real-time PCR. HFF were infected with pp28-luciferase and treated with either GCV or dimer sulfone carbamate. Luciferase activity was determined in cell lysates of infected-treated cells and infected non-treated cells. DNA copy number was determined by real-time PCR in supernatants of infected-treated cells and infected non-treated cells 6 days post infection.

generated with the laboratory-adapted strain AD169 was applied successfully to both qualitative and semiquantitative applications [5]. Compared to the GFP-CMV system, the luciferase-CMV offers a highly accurate and quantitative assay which is simple and easy to perform. A limited evaluation of pp28 -luciferase CMV activity in the presence of GCV, acyclovir and papaverine, suggested its potential application for anti-viral screen [26].

In addition to recombinant viruses, reporter cell lines have been generated to screen for anti-CMV compounds [6,27]. In one such approach, using a luciferase reporter cell line, the promoter was activated by immediate early proteins; therefore compounds that inhibit CMV at later stages of infection cannot be evaluated with this system [6]. Since the pp28-luciferase virus is driven by the promoter of a true late CMV gene, which can only occur after DNA replication and the onset of transcription of late genes, it can be applied for screening of compounds that target steps prior to and during DNA replication. The pp28-luciferase system therefore has a much wider application for drug screening compared to the reported luciferase cell line [6].

Quantification of viral genomes by real-time PCR is generally proportional to production of virus particles [7]. Application of real-time PCR for in-vitro screening of antiviral compounds is attractive because the assay is rapid and highly-sensitive. However, compared to the luciferase assay, real-time PCR is more labor-intensive. DNA copy number measured in supernatants collected at 6 days post infection with Towne virus correlated with luciferase activity in cell lysates at 3 and 6 days post infection. For a clinical isolate, generally 10 days were required for quantification of DNA in cell lysates [18]. Recently, a real-time PCR assay of a conserved region in UL54 was performed in cell lysates four days following infection and treatment with compounds and showed a high correlation with plaque reduction assay [12]. Additional studies are needed to determine the best timing and compartment for performance of the real-time PCR assay.

Our study reveals late CMV protein expression highly correlates with the production of infectious progeny (plaque assay) and DNA replication. Advantages of the luciferase assay over the real-time PCR include: faster turn-around time after infection, and lower cost (20 times less than real-time PCR). The luciferase assay yielded similar data to the plaque assay, but its performance (accuracy and rapidity) was superior. In conclusion, the recombinant pp28-lucifarese fulfills important characteristics that are required for high-throughput screening of anti-viral compounds: rapidity, reproducibility, low cost, and high sensitivity.

\section{Additional material}

Additional file 1: Sequences of the pp28, POL promoters and luciferase in the region between US9 and US10. Several regions can be distinguished- bold sequences are of CMV Towne, underlined sequences are POL (sequence \#1) and pp28 (sequence \#2) promoters, and the italic regions are the sequence of firefly luciferase gene.

\section{Abbreviations}

CMV: Cytomegalovirus; PCR: polymerase chain reaction; $\mathrm{EC}_{50}$ : effective concentration 50; HEL: human embryonic lung fibroblasts; HFF: human foreskin fibroblasts; MOI: multiplicity of infection; US: unique short; POL: polymerase. 
Acknowledgements

Supported by NIH KO8 Al074907 to RAB.

\section{Author details}

'Department of Pediatrics, Johns Hopkins University School of Medicine, Baltimore, MD, USA. ${ }^{2}$ The Sidney Kimmel Comprehensive Cancer Center, Johns Hopkins University School of Medicine, Baltimore, MD, USA.

${ }^{3}$ Department of Chemistry, School of Arts and Sciences, The Johns Hopkins University, Baltimore, MD, USA. ${ }^{4}$ Department of Pathology, Johns Hopkins Medical Institutions, Baltimore, MD, USA.

\section{Authors' contributions}

$\mathrm{RH}$ carried out the plaque/luciferase assays and verification of viral constructs. He participated in drafting the manuscript. GS, GSH and WHB designed and constructed the luciferase viruses, GHP synthesized and provided artemisinin derivatives, MF carried out the real-time PCR assays, RAB directed the study, analyzed and interpreted the data, drafted and revised the manuscript. All authors read and approved the manuscript.

\section{Competing interests}

The authors declare that they have no competing interests.

Received: 21 December 2010 Accepted: 26 January 2011

Published: 26 January 2011

\section{References}

1. Fishman JA, Emery V, Freeman R, Pascual M, Rostaing L, Schlitt HJ, Sgarabotto D, Torre-Cisneros J, Uknis ME: Cytomegalovirus in transplantation - challenging the status quo. Clin Transplant 2007, 21:149-158.

2. Kenneson A, Cannon MJ: Review and meta-analysis of the epidemiology of congenital cytomegalovirus (CMV) infection. Rev Med Virol 2007, 17:253-276.

3. Chou S: Cytomegalovirus UL97 mutations in the era of ganciclovir and maribavir. Rev Med Virol 2008, 18:233-246.

4. Kohler CP, Kerry JA, Carter M, Muzithras VP, Jones TR, Stenberg RM: Use of recombinant virus to assess human cytomegalovirus early and late promoters in the context of the viral genome. J Virol 1994, 68:6589-6597.

5. Marschall M, Freitag M, Weiler S, Sorg G, Stamminger T: Recombinant green fluorescent protein-expressing human cytomegalovirus as a tool for screening antiviral agents. Antimicrob Agents Chemother 2000, 44:1588-1597.

6. Fukui Y, Shindoh K, Yamamoto Y, Koyano S, Kosugi I, Yamaguchi T, Kurane I, Inoue N: Establishment of a cell-based assay for screening of compounds inhibiting very early events in the cytomegalovirus replication cycle and characterization of a compound identified using the assay. Antimicrob Agents Chemother 2008, 52:2420-2427.

7. Boeckh M, Boivin G: Quantitation of cytomegalovirus: methodologic aspects and clinical applications. Clin Microbiol Rev 1998, 11:533-554.

8. Boeckh M, Huang M, Ferrenberg J, Stevens-Ayers T, Stensland L, Nichols WG, Corey L: Optimization of quantitative detection of cytomegalovirus DNA in plasma by real-time PCR. J Clin Microbiol 2004, 42:1142-1148.

9. Hadaya K, Wunderli W, Deffernez C, Martin PY, Mentha G, Binet I, Perrin L, Kaiser L: Monitoring of cytomegalovirus infection in solid-organ transplant recipients by an ultrasensitive plasma PCR assay. J Clin Microbiol 2003, 41:3757-3764.

10. Gerna G, Furione M, Baldanti F, Percivalle E, Comoli P, Locatelli F: Quantitation of human cytomegalovirus DNA in bone marrow transplant recipients. Br J Haematol 1995, 91:674-683.

11. Lilleri D, Baldanti F, Gatti M, Rovida F, Dossena L, De Grazia S, Torsellini M, Gerna G: Clinically-based determination of safe DNAemia cutoff levels for preemptive therapy or human cytomegalovirus infections in solid organ and hematopoietic stem cell transplant recipients. J Med Virol 2004, 73:412-418.

12. Schnepf N, Boiteau N, Petit F, Alain S, Sanson-Le Pors MJ, Mazeron MC: Rapid determination of antiviral drug susceptibility of human cytomegalovirus by real-time PCR. Antiviral Res 2009, 81:64-67.
13. Kerry JA, Priddy MA, Kohler CP, Staley TL, Weber D, Jones TR, Stenberg RM: Translational regulation of the human cytomegalovirus pp28 (UL99) late gene. J Virol 1997, 71:981-987.

14. Jones TR, Muzithras VP, Gluzman Y: Replacement mutagenesis of the human cytomegalovirus genome: US10 and US11 gene products are nonessential. J Virol 1991, 65:5860-5872.

15. Jones TR, Muzithras VP: Fine mapping of transcripts expressed from the US6 gene family of human cytomegalovirus strain AD169. J Virol 1991, 65:2024-2036.

16. Lafemina RL, Hayward GS: Replicative forms of human cytomegalovirus DNA with joined termini are found in permissively infected human cells but not in non-permissive Balb/c-3T3 mouse cells. J Gen Virol 1983, 64(Pt 2):373-389.

17. Graham FL, van der Eb AJ: A new technique for the assay of infectivity of human adenovirus 5 DNA. Virology 1973, 52:456-467.

18. Arav-Boger R, He R, Chiou CJ, Liu J, Woodard L, Rosenthal A, JonesBrando L, Forman M, Posner G: Artemisinin-derived dimers have greatly improved anti-cytomegalovirus activity compared to artemisinin monomers. PLoS One 2010, 5:e10370.

19. Rosenthal AS, Chen X, Liu JO, West DC, Hergenrother PJ, Shapiro TA, Posner GH: Malaria-infected mice are cured by a single oral dose of new dimeric trioxane sulfones which are also selectively and powerfully cytotoxic to cancer cells. J Med Chem 2009, 2:1198-1203.

20. Tanaka Y, Kanda Y, Kami M, Mori S, Hamaki T, Kusumi E, Miyakoshi S, Nannya Y, Chiba S, Arai Y, Mitani K, Hirai H, Mutou Y: Monitoring cytomegalovirus infection by antigenemia assay and two distinct plasma real-time PCR methods after hematopoietic stem cell transplantation. Bone Marrow Transplant 2002, 30:315-319.

21. Stinski MF: Sequence of protein synthesis in cells infected by human cytomegalovirus: early and late virus-induced polypeptides. J Virol 1978, 26:686-701.

22. Vieira J, Schall TJ, Corey L, Geballe AP: Functional analysis of the human cytomegalovirus US28 gene by insertion mutagenesis with the green fluorescent protein gene. J Virol 1998, 72:8158-8165.

23. Wentworth $B B$, French L: Plaque assay of cytomegalovirus strains of human origin. Proc Soc Exp Biol Med 1970, 135:253-258.

24. Hippenmeyer PJ, Dilworth VM: A rapid assay for determination of antiviral activity against human cytomegalovirus. Antiviral Res 1996, 32:35-42.

25. Chou S, Van Wechel LC, Lichy HM, Marousek GI: Phenotyping of cytomegalovirus drug resistance mutations by using recombinant viruses incorporating a reporter gene. Antimicrob Agents Chemother 2005, 49:2710-2715.

26. Song $B H$, Lee $G C$, Lee $C H$ : Measurement of antiviral activities using recombinant human cytomegalovirus. The Journal of Microbiology 2000, 38:255-259.

27. Gilbert $\mathrm{C}$, Boivin $\mathrm{G}$ : New reporter cell line to evaluate the sequential emergence of multiple human cytomegalovirus mutations during in vitro drug exposure. Antimicrob Agents Chemother 2005, 49:4860-4866.

28. Freitas VR, Fraser-Smith EB, Matthews TR: Increased efficacy of ganciclovir in combination with foscarnet against cytomegalovirus and herpes simplex virus type 2 in vitro and in vivo. Antiviral Res 1989, 12:205-212.

29. Efferth T, Marschall M, Wang X, Huong SM, Hauber I, Olbrich A, Kronschnabl M, Stamminger T, Huang ES: Antiviral activity of artesunate towards wild-type, recombinant, and ganciclovir-resistant human cytomegaloviruses. J Mol Med 2002, 80:233-242.

30. Mercorelli B, Muratore G, Sinigalia E, Tabarrini O, Biasolo MA, Cecchetti V, Palu G, Loregian A: A 6-aminoquinolone compound, WC5, with potent and selective anti-human cytomegalovirus activity. Antimicrob Agents Chemother 2009, 53:312-315.

doi:10.1186/1743-422X-8-40

Cite this article as: $\mathrm{He}$ et al:: Recombinant luciferase-expressing human cytomegalovirus (CMV) for evaluation of CMV inhibitors. Virology Journal $20118: 40$. 